\title{
OPHTHALMOLOGY IN THE FACTORY
}

\author{
BY
}

IDA MANN

Oxford

Since vision is the dominant sense in man and since the majority of industrial processes are visual, it follows that ophthalmic questions are constantly arising in factory work. On entry, the problem of ocular fitness for the contemplated job should be considered: once the job has started the conditions of illumination are of importance, as is the problem of time lost from eye strain, which might have been prevented by the provision of adequate optical aids. Certain jobs carry certain eye risks, either of accident or of disease, and means of reducing these must be evolved. Their treatment, should they occur, is of importance. It must also be remembered and reiterated that a worker may suffer from an eye disease having no connexion with his work and that loss of time may, in many instances, be avoided by early diagnosis. Finally, should an accident or industrial disease occur, the questions of compensation, of traumatic neurasthenia and of rehabilitation, re-training and re-settlement will have to be dealt with.

The eye can be regarded from two aspects, either as an optical instrument or as a living organ. From the first point of view, it is often imperfect, but can be rendered efficient by mechanical means. From the second point of view, it can participate in general diseases of the body or can itself become diseased, showing, like any other tissues, changes which may be traumatic, inflammatory, neoplastic or degenerative. Finally, it must be realized that examination of an eye which is itself not producing symptoms, may lead to diagnosis of important disease in other organs, for example, the brain, pancreas or kidney. It is therefore obvious that examination of the eyes of factory workers at any stage should be carried out by an ophthalmic surgeon rather than by a non-medical refractionist, though the latter might very profitably work under the supervision of the former in order to save time. Since about 98 per cent. of the population show some error of refraction it is obvious that it is not necessary to correct every error that is found. The additional medical knowledge which the ophthalmic surgeon possesses over the refractionist enables him to assess the relation between the symptoms and the refractive error and so to decide whether spectacles are necessary in a given case. Many persons, for example, show a low grade hypermetropic astigmatism. If they are young, healthy, and have good binocular balance there is no need to correct the error, though the same error occurring in a person of middle age employed on fine work would seriously handicap him and would necessitate spectacles. Conversely, a low grade myopia is an advantage in middle age and even in young persons often need not be corrected. Figures given by Kuhn ${ }^{1}$ for occurrence of visual defects in industry are: defects in visual acuity 33 per cent., in depth perception 16 per cent., in colour perception 5 per cent., and in muscle balance 22 per cent. This was based on 16,332 examinations of workers already in jobs. Not * A post-graduate lecture given at the London School of Hygiene
and Tropical Medicine on May 13,1945 .

Industrial Ophthalmology. The C. V. Mosby Co., St. Louis, 1944. all of these, of course, need treatment, though many would be improved by it.

On entry into the factory, the workers should, ideally, have an eye examination which should include a test of visual acuity with and without correction of errors of refraction, a test of binocular balance and range of accommodation, an examination of the fundus and a rough estimate of the field of vision. In this way one will gain an idea of the type of work which can be undertaken and will avoid the obvious pitfalls of putting uncorrected presbyopes on to precision or clerical work, or myopes on to long-range jobs. Such counsel is that of perfection and may well be unattainable. Even if, however, a full test cannot be carried out an attempt should be made to test the acuity at the distance of the proposed job. Some factories, following the example of the Royal Navy and the railways, give a 6-metre test only and consider that a visual acuity of $6 / 36$ or $6 / 60$ is undesirable, in spite of the fact that, if it is caused by a low grade myopia, it may well be an advantage in precision work. This led, not so long ago, to the naïve statement by an American factory that they were surprised to find on analysis that the men with the worst visual acuity for distance did the best fine work and complained the least of eye strain. A recent investigation $^{2}$ of a group of workers on a very close job (spiral filament inspection) brought out the fact, long suspected by ophthalmologists, that perfect binocular balance at the near point is of more importance in the avoidance of eye strain than is perfect correction of refractive errors. The mere provision of spectacles will, therefore, do little to relieve the precision worker, since routine correction of refraction will lead to anomalies such as correction of low grade myopia in early middle age, or correction of hypermetropia in young exophorics with convergence deficiency. It is better to be entirely monocular than imperfectly binocular, a fact which may account for the almost universal adoption of the watchmaker's monocular loupe rather than any binocular device of similar power but more difficult adjustment. When, however, an individually prescribed binocular loupe can be obtained, greater comfort and often improved visual acuity will ensue owing to retention of binocular vision and depth perception. In some trades, however, the magnification required may be too great for comfort with a binocular loupe and a short monocular working distance with a higher magnification may be found preferable. Each job should be separately studied and visually assessed. It is quite possible by a study of the requirements of the job and of the worker's eyes to prescribe in every case (disease apart) a suitable optical instrument, monocular, binocular, or bifocal and prismatic, to enable him to do that job in comfort. This type of prescribing requires ingenuity and a study of conditions on the spot. It has been tried in the United States of America and advocated and described in Kuhn's book on industrial ophthalmology.

In addition to his individual industrial prescribing, the ophthalmologist should satisfy himself that the con-

2 Mann, I., and Archibald, D. (1944). Brit. med. J., 1, 387. 
ditions of illumination are satisfactory. Glare should be avoided, shadowless lights should not be used where grain and texture are to be observed, fluorescent tubes are often unsuitable if the work entails rapid side to side movements and there should never be too great a contrast between the illumination of the work and of the rest of the room.

The question of periodic eye examination while on the job now arises. Should the factory be responsible for this, as the railway companies already are? The answer is that it should be responsible if possible, since we know that latent hypermetropia becomes slowly manifest and a job suitable for a hypermetropic boy may lead to eyestrain at 30 if he is not given glasses at the right time. The age of onset of presbyopia varies slightly and is, of course, greatly altered by the initial refraction, but on the whole one can say that a sight test every three or five years would save a fair proportion of discomfort. Annual tests, obvious disease apart, are not necessary, but certain insidious diseases such as glaucoma, may be missed in the later age groups if the interval is too long.

Having put and maintained our workers in the best conditions to do the job, we must now deal with industrial risks, such as risks from flying particles, risks from chemical fluids and vapours and risks from irradiation. Here again the aim should be prevention. Possibly the greatest ophthalmic need in industry to-day is the production and popularization of a comfortable and efficient protective goggle and the establishment of a 'goggle maintenance service,' such as is used in certain American factories. The problem has been grossly neglected in this country, and the number of minor eye injuries, which are preventable, is very great.

If the injury has not been prevented, we are faced with its treatment. If a foreign body is small and superficial it can be dealt with by a nurse (provided she has worked in an eye hospital) or by the factory doctor. It must be carefully removed with the minimum of damage. The question of prevention of sepsis is one which is receiving much attention at present. Experimental work points to sulphonamide or penicillin as the best prophylactic, but it is not yet certain that either is of any use as a single application. The present 'factory' eye drops (containing cocaine and hydrarg. perchlor. in castor oil) are of doubtful value and if misapplied may do harm. They usually produce intense discomfort.

Patients with more serious injuries should be sent to hospital. Small inconspicuous wounds of the cornea in which the foreign body cannot be seen are the most dangerous, as they probably mask an intraocular foreign body. Injuries in the ciliary region and to the lens are the most serious. All patients with injuries suspected of perforating are surgical emergencies and must be sent to an eye hospital at once.

The industrial eye diseases peculiar to the factory involved should, of course, be especially studied by the medical officer. Such things as welder's flash, chemical burns, reactions to irritants, and 'fumed eyes' in viscose factories, all require their special prophylaxis and treatment. Welder's flash is a superficial radiational burn of the corneal epithelium. It is not apparent at the moment it happens and perhaps more often results from the striking of an are by someone other than the patient, since all welders, after one experience, are careful to protect themselves from their own arc. The symptoms are an intense irritation, lacrimation and pain coming on about six hours after exposure. If this is in the night the patient will be awakened by the pain. If it comes on during working hours drops of cocaine and ephedrin are indicated for the relief of pain. Healing is usually rapid.
Chemical burns are more serious. Acid burns on the whole recover more quickly and leave less disability than do alkali burns, which lead to intense oedema and may take months to subside. Other more complicated substances may cause ocular injury by splash or vapour, such as the various materials used in chemical warfare. Various types of acute and chronic keratitis can be recognized, as, for example, that caused by di-chlorodiethyl sulphide (mustard gas). This produces in severe cases a vascularizing keratitis which partially recovers and is then subject to repeated re-ulceration, even many years after the exposure. Injuries by di-vinyl arsine on the other hand give destructive lesions which once healed do not break down. Specific antidotes to be applied at once exist for some compounds, but copious irrigation with a large volume of water is often of great value as a first aid treatment.

In some trades there is a specific risk, for example, 'fumed eyes.' This is found in viscose workers in factories manufacturing 'silk' stockings where the worker is inadequately protected from the microscopic spray (probably of varying concentrations of $\mathrm{H}_{2} \mathrm{SO}_{4}$ ) shaken from the spun thread as it leaves the spinning bath to be wound on the spindle. The symptoms, discomfort and the appearance of coloured haloes round lights, are caused by a fine oedema of the superficial cells of the corneal epithelium. This disappears in a day or two if the worker is not further subjected to the spray. Other processes carry a long term risk, such as the risk of cataract in glass blowers and chain makers, or of nystagmus in miners. Both these conditions are largely preventable, the former by the use of Infrex or other glass goggles which cut out infra red rays, the latter by more efhcient illumination and improved conditions in the mines.

We must now return to the question of the occurrence of non-industrial eye disease in the factory. Prompt attention to any red or painful eye may save much lost time from iritis or glaucoma and early recognition of contagious conditions (e.g. Koch-Weeks conjunctivitis and epidemic kerato-conjunctivitis) may save spread throughout a shop or the whole factory. Differential diagnosis between acute conjunctivitis, acute iritis and acute glaucoma may save many serious complications. It is, however, a difticult diagnosis to make without the help of an ophthalmic surgeon. It is always better to suspect iritis or glaucoma when it is not present than to miss it when it is. An acute iritis treated promptly (the first day) with atropine will often be well in a week or so, but if neglected (i.e. treated as a conjunctivitis) for a week may necessitate admission to hospital, operation, and prolonged or even permanent disability. A wronglytreated glaucoma involves an even greater risk to the patient.

The question of general disease affecting the cyes of workers is also of importance. For example, a large number of cases of diabetes are diagnosed in the first instance in eye hospitals, where the patients have come for a change of glasses. Albuminuria, arterio-sclerosis, cerebral tumour, various vitamin deficiencies and anaemias, as well as syphilis, tuberculosis and sarcoidosis have definite diagnostic eye signs, often in stages where there is little or no general disturbance. Periodic examination of workers would detect a certain number of these.

That the factory ophthalmic service shall be as efficient as that outlined here is probably a counsel of perfection, but factory doctors with partial training in ophthalmology can do much by careful selection of cases for further opinion and examination. 